\title{
A new synthesis route to prepare polyaniline (PANI) nanotubes containing magnetic nanoparticles
}

\author{
De Araújo A. C. V. ${ }^{1, a}$, Alves Jr. S. ${ }^{1, b}$ and Azevedo W. M. ${ }^{1, c}$ \\ ${ }^{1}$ Universidade Federal de Pernambuco UFPE, Centro de Ciências Exatas e da Natureza, \\ Departamento de Química Fundamental, sem número, CEP: 50970-901, Recife, PE, Brazil \\ aacva@nlink.com.br, ${ }^{b}$ salvesjr@ufpe.br, ${ }^{\text {cwma@ufpe.br }}$
}

Keywords: nanoparticles, iron oxide, nanocomposite, polyaniline.

\begin{abstract}
In this work we report the preparation and characterization of a polyaniline/magnetite (PANI) $-\mathrm{Fe}_{3} \mathrm{O}_{4}$ nanocomposite, with average diameter around $50 \mathrm{~nm}$ and tubular morphology. The tubular nanocomposite was synthesized by an in situ polymerization of aniline using $\mathrm{Fe}_{3} \mathrm{O}_{4}$ nanoparticles as an oxidant agent. The $\mathrm{Fe}_{3} \mathrm{O}_{4}$ nanoparticles with narrow size distribution were synthesized by co-precipitation technique and the products were characterized by powder X-ray diffractometry (XRD), Fourier transform infrared (FTIR), scanning electron microscopy (SEM), and transmission electron microscopy (TEM).
\end{abstract}

\section{Introduction}

Polymer-inorganic composites with an organized structure provide a new functional hybrid between organic and inorganic materials [1, 2]. Among these polymers, polyaniline (PANI) has attracted particular interest due to the fact that its electrical properties can be reversibly controlled by changing the oxidation state of the main chain and by protonating the imines nitrogen atoms [1].

A considerable number of articles have been published on the magnetic and conducting polymeric nanocomposites of polyaniline (PANI) as well as polypyrrole composites containing nanoparticles such as $\mathrm{TiO}_{2}, \mathrm{ZrO}_{2}, \mathrm{Fe}_{2} \mathrm{O}_{3}, \mathrm{Fe}_{3} \mathrm{O}_{4}$ and $\mathrm{SnO}_{2}$. The properties of these systems are sensitive to the particle size, inter particle interaction, and temperature [3]. Wan et al. studied a series of PANI composites containing nanomagnets prepared by chemical polymerization [4]. Deng et al. reported the preparation of PANI- $\mathrm{Fe}_{3} \mathrm{O}_{4}$ nanoparticles with core-shell structure via an in situ polymerization of aniline monomer in an aqueous solution, which contains $\mathrm{Fe}_{3} \mathrm{O}_{4}$ nanoparticles and surfactant NaDS [5]. The reason for that seems to be the fact that they have many potentials applications in electromagnetic interference shielding [6], electrochromics device [7] non-linear optical systems [8].

Since the discovery of carbon nanotubes by Iijima [9], one-dimensional nanostructures of various materials including metal, sulfide, metal oxides, polymer and even composite have been a subject of intense research because of their potential applications in many areas. In recent years, nanostructures of PANI including nanowires, nonorods and nanotubes have been studied extensively. Few reports have studied the $1 \mathrm{D}$ nanostructures of PANI containing $\mathrm{Fe}_{3} \mathrm{O}_{4}$ nanoparticles. Wan et al. developed a self-assembly method to synthesize 1D PANI-Fe $\mathrm{O}_{4}$ nanostructures, however, the content of $\mathrm{Fe}_{3} \mathrm{O}_{4}$ is small [10]. In the recent work Alam et al [3] synthesized PANI/ $\mathrm{Fe}_{3} \mathrm{O}_{4}$ nanocomposites using ferrofluid to polymerize aniline monomer in solution and found that the polymer structure of the nanocomposite present a porous structure with two phase systems.

In this work, we report the results of a new synthetic route to obtain tubular nanocomposites of $\mathrm{PANI} / \mathrm{Fe}_{3} \mathrm{O}_{4}$ using $\mathrm{Fe}_{3} \mathrm{O}_{4}$ magnetic nanoparticles as oxidant agent to polymerize aniline monomer solution, the synthesis process was analyzed as a function of the reaction time and the kind of acid used to prepare the solution. In that case no extra oxidant is necessary for the synthetic process, in this method we putt the $\mathrm{Fe}_{3} \mathrm{O}_{4}$ nanoparticles in contact with the aniline acid solution under UV irradiation and after the respective time we obtained the nanocomposite with tubular nanostructures 
precipitation. The products were characterized by powder X-ray diffractometry (XRD), Fourier transform infrared (FTIR), scanning electron microscopy (SEM), and transmission electron microscopy (TEM).

\section{Experimental}

\section{Materials}

Aniline monomer (Vetec, 99\%) was double distilled under reduced pressure and stored at low temperature before use. Iron (II) sulfate heptahydrate $\left(\mathrm{FeSO}_{4} .7 \mathrm{H}_{2} \mathrm{O}\right)$ (Vetec, $99 \%$ ), sodium hydroxide $(\mathrm{NaOH})$ (Riedel-de Haën, 99\%), nitric acid $\left(\mathrm{HNO}_{3}\right)$ (Dinâmica, 65\%), were all of analytical reagent grade and used as received.

\section{Synthesis}

The magnetic nanoparticles of $\mathrm{Fe}_{3} \mathrm{O}_{4}$ were obtained from a suspension of $\mathrm{FeSO}_{4} .7 \mathrm{H}_{2} \mathrm{O}$ under sonication, where a solution of $\mathrm{NaOH}$ was added. This mixture was left under ultrasound for $1 \mathrm{~h}$. After that a black magnetic precipitate $\left(\mathrm{Fe}_{3} \mathrm{O}_{4}\right)$ was obtained. The precipitate was washed several times with chloride acid solution, centrifuged and then dried under rotaevaporation.

To these nanoparticles a nitrite aniline solution was added, and was left under UV radiation for $1,2,3$ or $4 \mathrm{~h}$, as the polymerization starts the suspension turned into a dark green which proves the aniline polymerization is taking place. The composite was centrifuged, washed with distilled water, acetonitrile and dried under vacuum.

\section{General Instrumentation}

Wide angle X-ray diffraction patterns of the samples were colleted on a Rigaku diffractometer model DMAX 2400, using $\mathrm{Cu} \mathrm{K} \alpha$ radiation $(\lambda=0.15418 \mathrm{~nm})$ at a scanning speed of $0,1^{0} \mathrm{~s}^{-1}$ in the range of $2 \theta=15-80^{0}$ with a step $0,02^{0}$. The average crystallite size was estimated for the integral intensity of the X-ray diffraction peak (311) using the Scherer equation 1 :

$$
T c=k \lambda / \alpha \cos \theta
$$

where $k$ is the shape factor, $\lambda$ is the $\mathrm{X}$-ray wavelength $(0.15418 \mathrm{~nm}), \alpha$ is the full width at halfmaximum expresses in until of $2 \theta$, and is the Bragg angle $(0)$.

The FT-IR spectra of the products were analyzed on a Bruker model IFS-66 in the range of $4000-400 \mathrm{~cm}^{-1}$ using $\mathrm{KBr}$ pellets.

The morphology was measured by a scanning electron microscope (SEM, JSM-5900, Joel instruments, Japan) and a transmission electron microscope (TEM, Tecnai-FEI).

\section{Results and Discussion}

Fig. 1 shows the wide angle X-ray diffraction patterns for the (a) $\mathrm{Fe}_{3} \mathrm{O}_{4}$ pure, (b) $\mathrm{PANI} / \mathrm{Fe}_{3} \mathrm{O}_{4}$ nanocomposites obtained after 1 hour of UV irradiation, (c) $\mathrm{PANI} / \mathrm{Fe}_{3} \mathrm{O}_{4}$ nanocomposites obtained after 2 hours of UV irradiation, (d) $\mathrm{PANI} / \mathrm{Fe}_{3} \mathrm{O}_{4}$ nanocomposites obtained after 3 hours of UV irradiation, (e) $\mathrm{PANI} / \mathrm{Fe}_{3} \mathrm{O}_{4}$ nanocomposites obtained after 4 hours of UV irradiation and (f) PANI pure. For $\mathrm{Fe}_{3} \mathrm{O}_{4}$ sample, a single spinel phase structure is assigned with the characteristic reflections of the Fd3m cubic spinel group. Using the Debye-Scherrer equation analysis the average crystallite size $(T c)$ can be estimated to be $25.38 \mathrm{~nm}$.

Fig. 1 (b-e) shows the X-ray diffraction patterns for the composites $\mathrm{PANI} / \mathrm{Fe}_{3} \mathrm{O}_{4}$, synthesized in nitric acid, after UV irradiation for 1, 2, 3 or 4 hours. It is observed that the crystal size diameter decrease with the increase of the interaction time with UV light with the solution. The average crystallite size, $T c$, for $1,2,3$ and 4 hours the interaction of interaction with UV light was 24.20; 
24.97; 25.15 and $19.91 \mathrm{~nm}$, respectively. After 4 hours it is observed that $T c$ decreases about $6 \mathrm{~nm}$, and a new phase is formed, probably it can assigned as being $\gamma-\mathrm{Fe}_{2} \mathrm{O}_{3}$ (JCPDS n $\mathrm{n}^{0}$ : 52-1449).

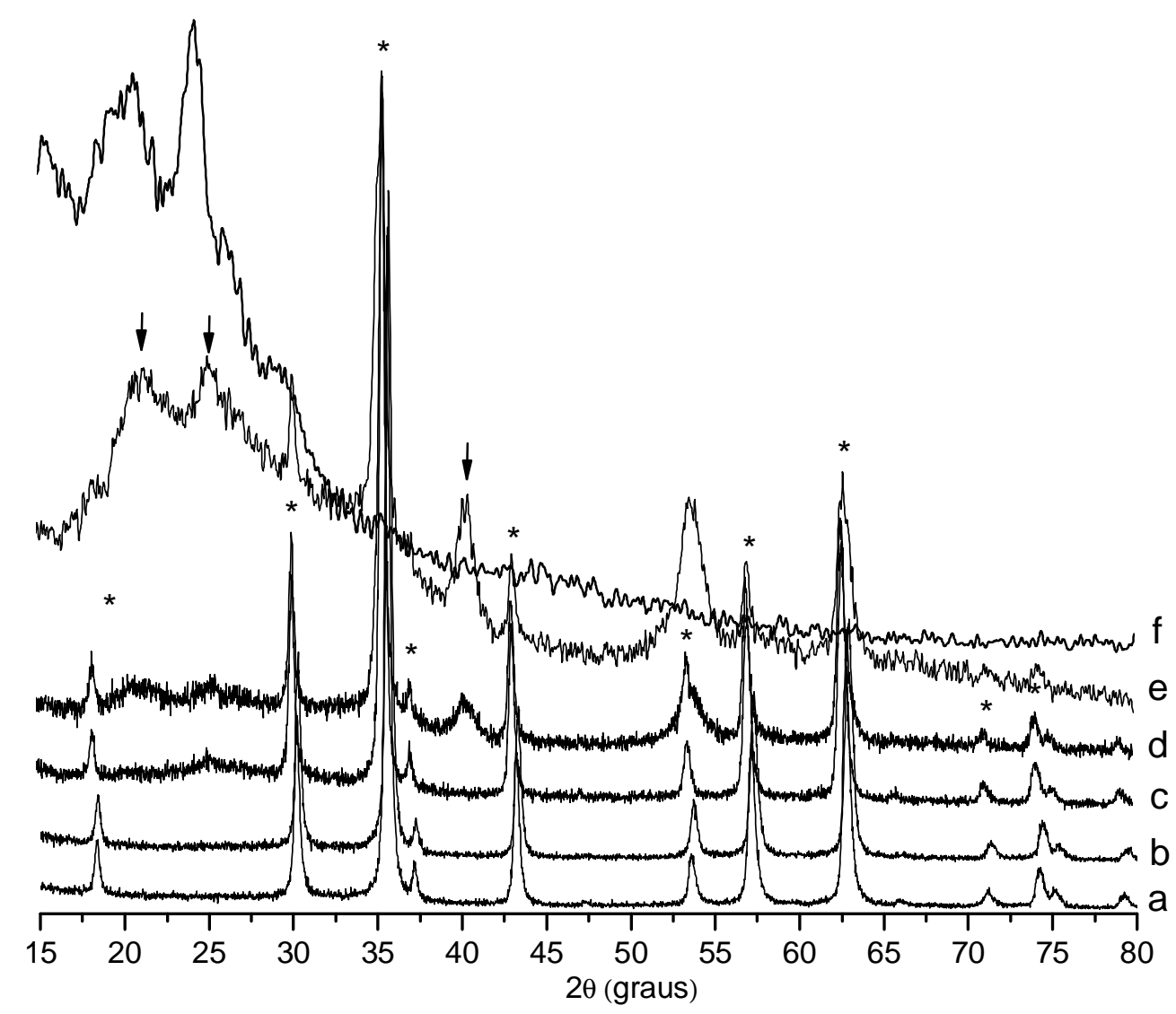

Fig 1: X-ray diffraction patterns for the (a) $\mathrm{Fe}_{3} \mathrm{O}_{4}$ pure, (b) $\mathrm{PANI} / \mathrm{Fe}_{3} \mathrm{O}_{4}$ nanocomposites obtained after 1 hour of UV irradiation, (c) PANI/ $/ \mathrm{Fe}_{3} \mathrm{O}_{4}$ nanocomposites obtained after 2 hours of UV irradiation, (d) $\mathrm{PANI} / \mathrm{Fe}_{3} \mathrm{O}_{4}$ nanocomposites obtained after 3 hours of UV irradiation, (e) $\mathrm{PANI} / \mathrm{Fe}_{3} \mathrm{O}_{4}$ nanocomposites obtained after 4 hours of UV irradiation and (f) PANI pure.

The FT-IR spectra analysis for pure magnetite, pure polyaniline and $\mathrm{PANI} / \mathrm{Fe}_{3} \mathrm{O}_{4}$ nanocomposites after 1, 2, 3 and $4 \mathrm{~h}$ of UV irradiation are shown in the figure 2. For the spectrum of the pure magnetite a small peak can be observed around $3200 \mathrm{~cm}^{-1}$ characteristic of the stretching O$\mathrm{H}$ that can be attributed to the water molecule adsorbed onto the surface of the magnetite nanoparticles, also peaks can be observed around 581, 796 e $889 \mathrm{~cm}^{-1}$ which can be attributed to the stretching vibration mode of Fe-O.

For pure PANI (fig. 2) the presence of benzenoid (NH-B-NH) and quinoid (NH-Q-NH) rings vibration is observed at $1500 \mathrm{~cm}^{-1}$ and $1574 \mathrm{~cm}^{-1}$, respectively, characterizing the oxidation state of emeraldine salt form of PANI. Two other modes can be observed at $1134 \mathrm{e} 1382 \mathrm{~cm}^{-1}$ and one mode around $800 \mathrm{~cm}^{-1}$ which can be aattributed to the stretching vibration mode $\mathrm{C}-\mathrm{N}$ and $\mathrm{C}-\mathrm{H}$, respectively, this results seems to be in agrement with the literature results for polyaniline in the emeraldine salt form.

For the PANI/Fe $\mathrm{F}_{3} \mathrm{O}_{4}$ nanocomposites we can see that the vibration modes of $\mathrm{Fe}_{3} \mathrm{O}_{4}$ is shifted to 595,799 e $877 \mathrm{~cm}^{-1}$ and the relative intensity of this mode is changed, characterizing a possibly structure modification during the polymerization process, this results seems to be in agreement with the $\mathrm{x}$-ray measurement where a new phase $\gamma-\mathrm{Fe}_{2} \mathrm{O}_{3}[\mathbf{1 1}$, can be observed after 4 hour of synthesis, 
also we can observed that a small shift in the polymer mode is observed, indicating a possibly interaction between polymer and the magnetic particles

These results seems to be a strong evidence of the reaction of the $\mathrm{Fe}_{3} \mathrm{O}_{4}$ nanoparticles with aniline solution in order to form the polymer/ $\mathrm{Fe}_{2} \mathrm{O}_{3}$ nanocomposite particle.

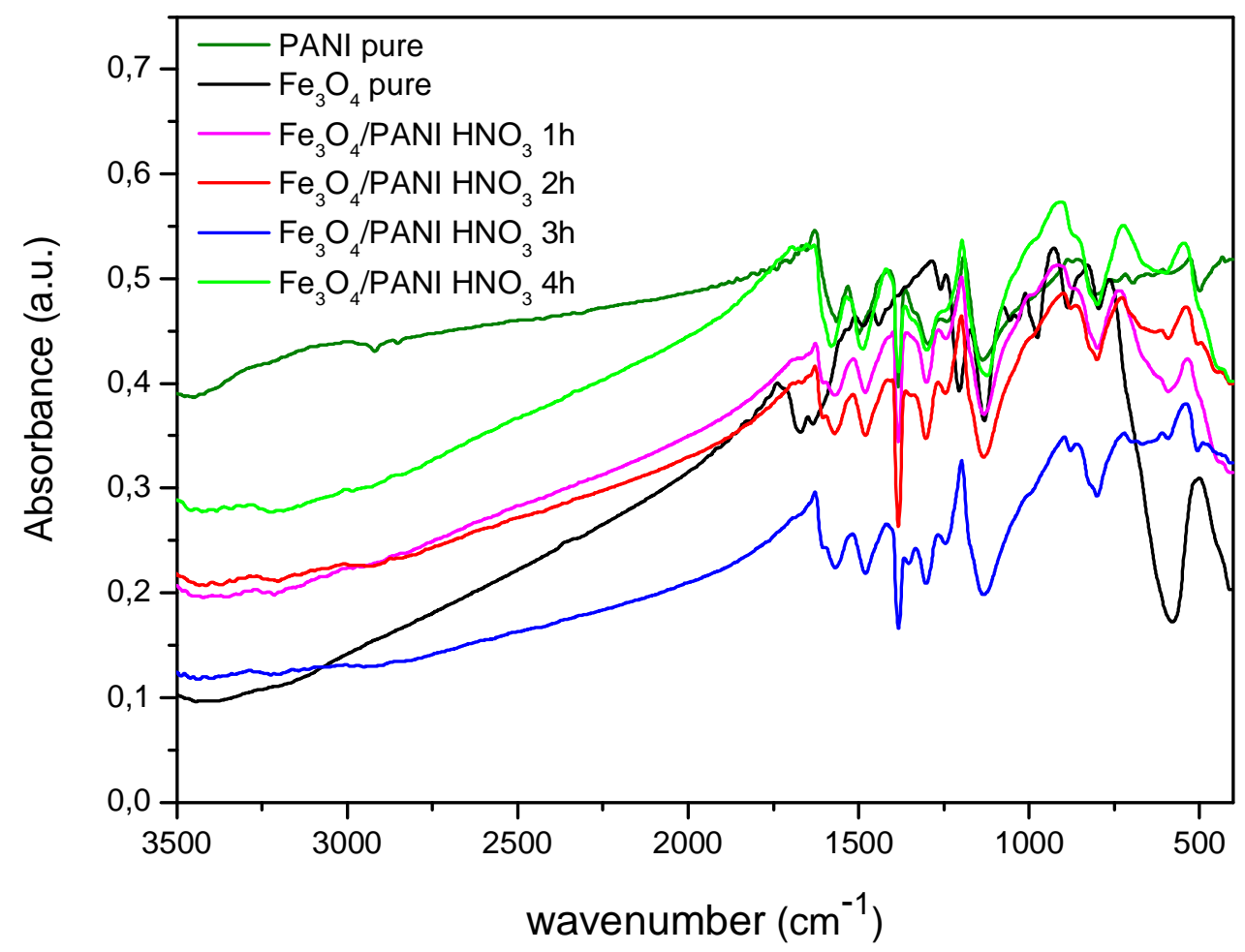

Fig. 2: FTIR spectra of PANI pure, $\mathrm{Fe}_{3} \mathrm{O}_{4}$ pure and $\mathrm{PANI} / \mathrm{Fe}_{3} \mathrm{O}_{4}$ composite after $1,2,3$ and $4 \mathrm{~h}$ with $\mathrm{UV}$ radiation.

Fig. 3 shows the SEM analyses for $\mathrm{Fe}_{3} \mathrm{O}_{4}$ present a ball like shape with average diameter of $73,05 \pm 25,56 \mathrm{~nm}$, indicating that small particle might agglomerate, to form $\mathrm{Fe}_{3} \mathrm{O}_{4}$ cluster due to magnetic interacting or inter molecular interaction from the X-ray analysis the means diameter of the particles was found to be of the order of $30,97 \mathrm{~nm}$.

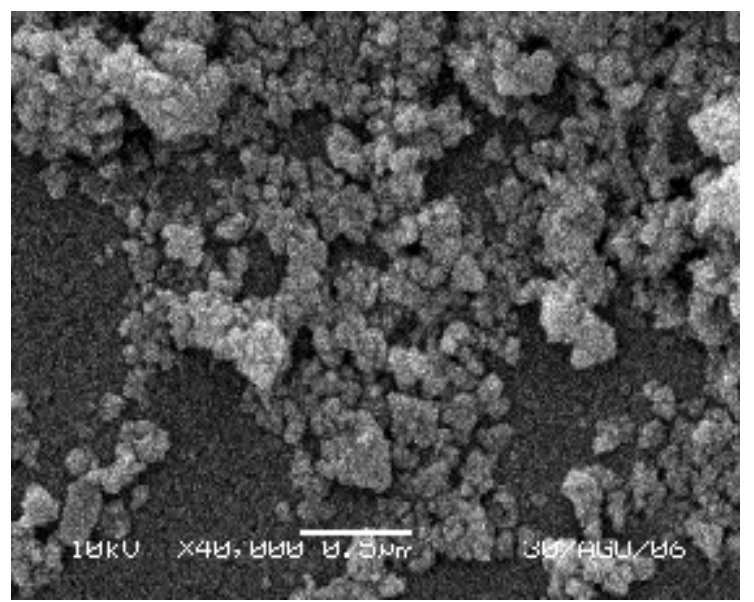

Fig. 3: SEM analysis for the $\mathrm{Fe}_{3} \mathrm{O}_{4}$ pure. 
The SEM images for PANI/Fe ${ }_{3} \mathrm{O}_{4}$ (Fig. 4) reveal that the composite presents a fiber shape with mean diameter of the order of 60-80 nm.
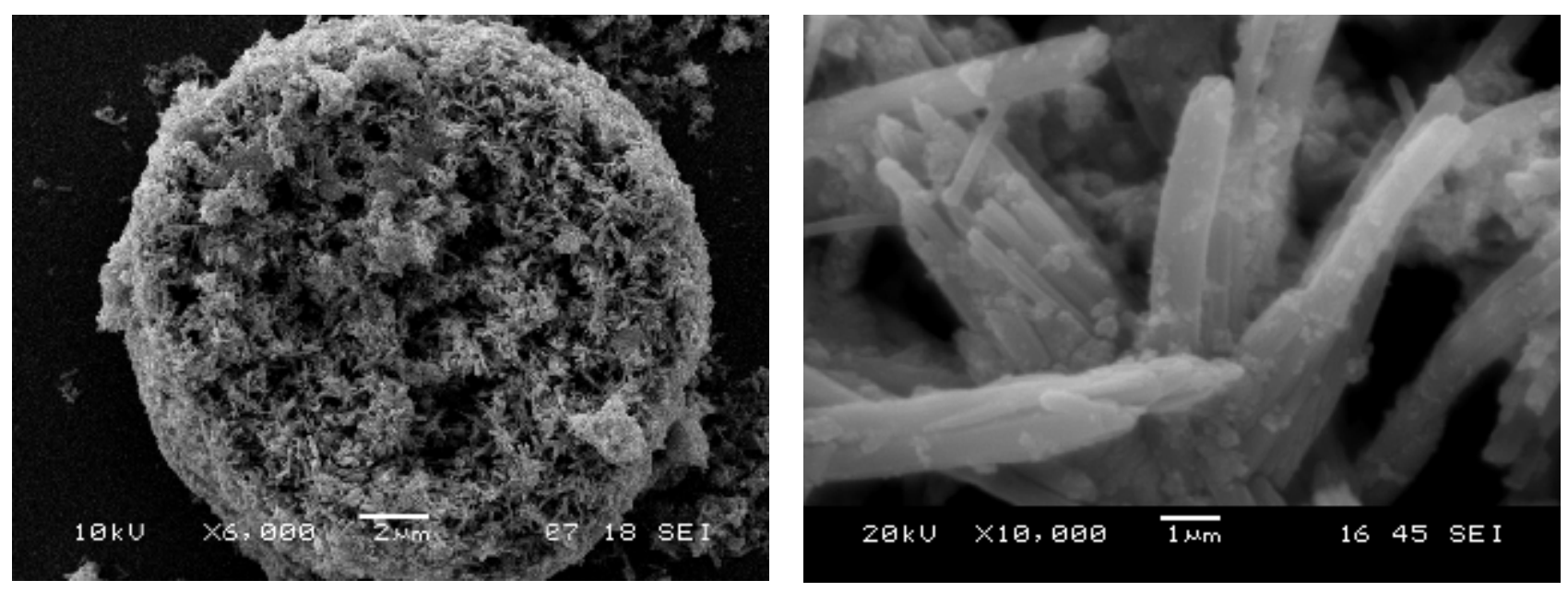

Fig. 4: Micrographies showing tubular morphology.

The TEM images (Fig. 5) suggest this hollow fiber as nanotube, but a small portion of them is solid as nanorod. This results seems to be consistent with Huang et al [12] results for PANI$\mathrm{NSA} / \mathrm{Fe}_{3} \mathrm{O}_{4}$ composite.
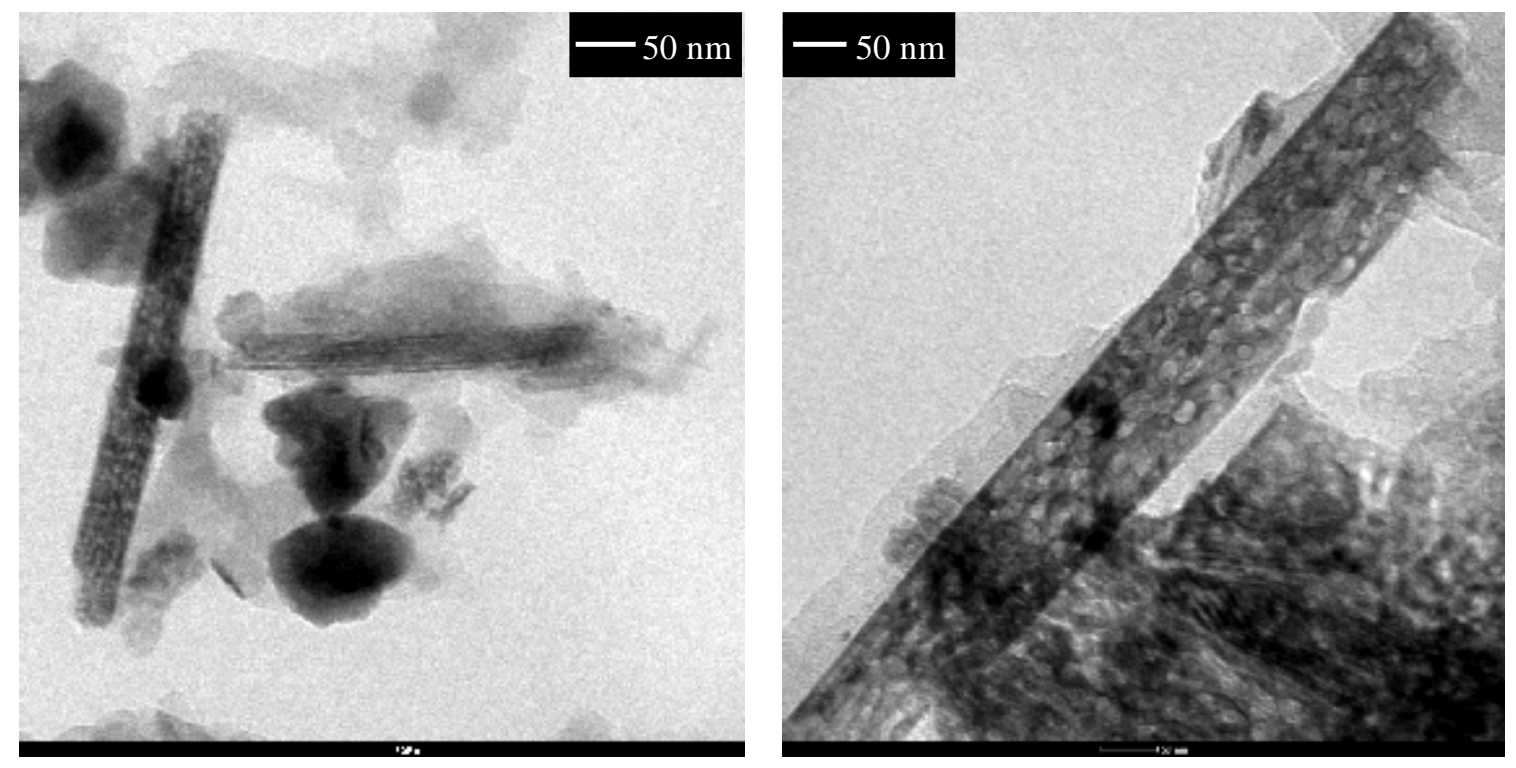

Fig. 5: TEM micrographies showing tubular morphology.

\section{Conclusions}

In this work we demonstrated a new method of synthesis of PANI nanofiber containing $\mathrm{Fe}_{3} \mathrm{O}_{4}$ nanoparticles that act as oxidizing agent for the aniline monomer in the polymerization process, under UV irradiation. SEM and TEM images showed the morphology of the nanofibrous composite and suggested that $\mathrm{Fe}_{3} \mathrm{O}_{4}$ nanoparticles may be embedded in the composites. 


\section{Acknowledgements}

The authors wish to acknowledge the financial support from the Brazilian agency CNPq (Conselho Nacional de Desenvolvimento Científico e Tecnológico) and RENAMI network. Also would like to thank Francisco Rangel and João Carlos César de Albuquerque for the SEM X-ray measurements.

\section{References}

[1] Falcão E. H. L., Azevedo W. M., Synthetic Metals 128 (2002) 149.

[2] Yoshimoto S., Ohashi F., Kameyama T., Macromol Rapid Commun. 25 (2004) 1687.

[3] Alam J., Riaz U., Ahmad S., J. of Magnetism and Magnetic Materials 314 (2007) 93.

[4] Wan M. X., Li W. C., J. Polym. Sci. Part A: Polym. Chem 34 (1997) 2129.

[5] Deng J. G., He C. L., Peng Y. X., Wang J. H., Long X. P., Li P., Chan A. S. C., Synth. Met. 139 (2003) 295.

[6] Miyauchi S., Aiko H, Sorimashi Y., Tsubata I., J. Appl. Polym. Sci. 37 (1989) 289.

[7] Shen P. K., Huang H. T., Tseung A. C. C., J. Electrochem. Soc., 139 (1992) 1840.

[8] Peng X., Zhang Y., Yang J., Zou B., Xia P. L. et al, J. Phys. Chem., 96 (1992) 3412.

[9] Iijima S., Nature 354 (1991) 56.

[10] Zhang Z. M., Wan M. X., Wei Y., Nanotechnology 16 (2005) 2827.

[11] Apte S. K., Naik S. D., Sonawane R. S., Kale B. B., J. Am. Ceram. Soc., 90 (2007) 412.

[12] Huang J., Wan M. X., J. Polym. Sci.: A polym. Chem.. 37 (1999) 151. 